\begin{tabular}{|c|c|}
\hline \multirow{3}{*}{ 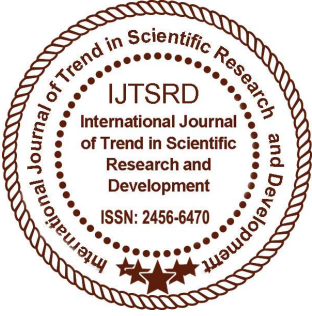 } & $\begin{array}{l}\text { International Journal of Trend in Scientific } \\
\text { Research and Development (IJTSRD) }\end{array}$ \\
\hline & International Open Access Journal \\
\hline & ISSN No: 2456 - 6470 | www.ijtsrd.com | Volume - 2 | Issue - 4 \\
\hline
\end{tabular}

\title{
Simulation of Direct Sequence Spread Spectrum for Wireless Communication Systems using Simulink
}

\author{
Y.V.S Durga Prasad \\ Associate Professor, ECE Dept, ACE Engineering \\ College, Hyderabad, Telangana, India
}

\author{
K. Venkateswarlu \\ Assistant Professor, ECE Dept, ACE Engineering \\ College, Hyderabad, Telangana, India
}

\section{ABSTRACT}

In this work, a simulation model for Direct Sequence Spread Spectrum (DSSS) scheme for wireless communication systems has been proposed. Unlike the case of a single frequency carrier, the modulated signal in DSSS occupies a much wider bandwidth in order to reduce the possible interferences with narrow band communication signals. In telecommunications, DSSS is a spread spectrum modulation technique used to reduce overall signal interference. The spreading of this signal makes the resulting wideband channel more noisy, allowing for greater resistance to unintentional and intentional interference.

Keywords: Code division multiple access, Direct Sequence Spread Spectrum, interference

\section{INTRODUCTION}

Spread spectrum communication systems are widely used today in a variety of applications for different purposes such as access of same radio spectrum by multiple users (multiple access), anti-jamming capability, interference rejection, secure communications, multi-path protection, etc. However, irrespective of the application, all spread spectrum communication systems satisfy the following criteria-

$>$ As the name suggests, bandwidth of the transmitted signal is much greater than that of the message that modulates a carrier.
The transmission bandwidth is determined by a factor independent of the message bandwidth.

Based on the kind of spreading modulation, spread spectrum systems are broadly classified as-

I. Direct sequence spread spectrum systems

II. Frequency hopping spread spectrum (FH-SS) systems

III. Time hopping spread spectrum systems

IV. (iv) Hybrid System

In DSSS-CDMA a random spreading code sequence $c(t)$ of chosen length is used to 'spread'(multiply) the modulating signal $\mathrm{m}(\mathrm{t})$. Sometimes a high rate pseudo-noise code is used for the purpose of spreading. Each bit of the spreading code is called a 'chip'. Duration of a chip (Tc) is much smaller compared to the duration of an information bit (T)..

Consider Binary Phase Shift Keying (BPSK) for modulating a carrier by this spread signal. If $\mathrm{m}(\mathrm{t})$ represents a binary information bit sequence and $\mathrm{c}(\mathrm{t})$ represents a binary spreading sequence, the 'spreading' or multiplication operation reduces to modulo-2 or ex-or addition. For example, if the modulating signal $\mathrm{m}(\mathrm{t})$ is available at the rate of 10 Kbits per second and the spreading code $\mathrm{c}(\mathrm{t})$ is generated at the rate of 1 Mbits per second, the spread signal $d(t)$ is generated at the rate of 1 Mega Chips per second. So, the null-to-null main lobe bandwidth of 
the spread signal is now $2 \mathrm{MHz}$. We say that bandwidth has been 'spread' by this operation by a factor of hundred. This factor is known as the spreading gain or process gain (PG).

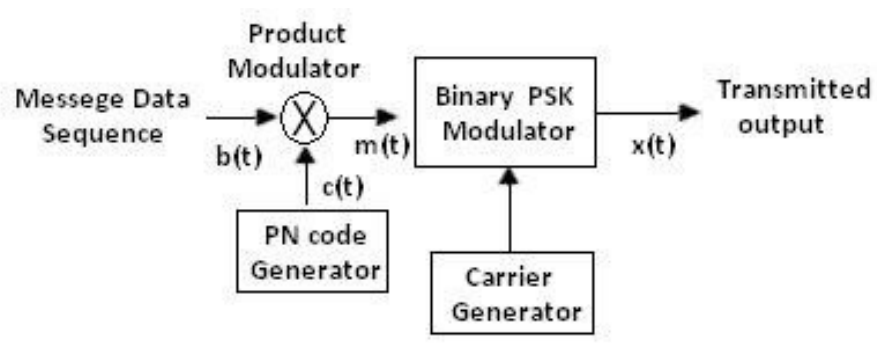

Transmitter

Fig below shows the baseband processing operations necessary after carrier demodulation. Note that, at the receiver, the operation of despreading requires the generation of the same spreading code incorrect phase with the incoming code. The pseudo noise (PN) code synchronizing module detects the phase of the incoming code sequence, mixed with the information sequence and aligns the locally generated code sequence appropriately. After this important operation of code alignment (i.e. synchronization) the received signal is 'despread' with the locally constructed spreading code sequence. The despreading operation results in a narrowband signal, modulated by the information bits only.

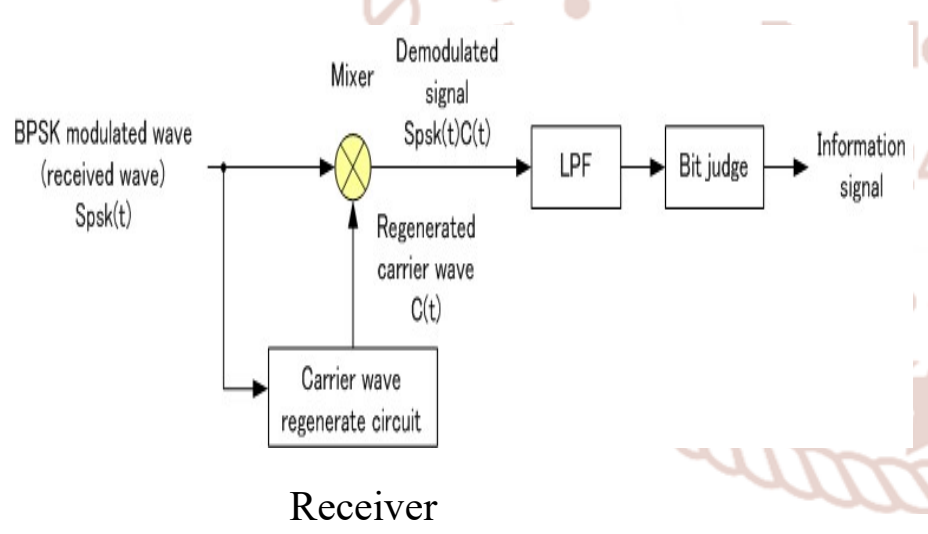

\section{DIRECT SEQUENCE SPREAD SPECTRUM features}

Each bit is represented by multiple bits using spreading code

- Spreading code spreads signal across wider frequency band - In proportion to number of bits used -e.g., 10 bit spreading code spreads signal across 10 times bandwidth of 1 bit code

- One method: - Combine input with spreading code using XOR
- Input bit 1 inverts spreading code bit

- Input zero bit doesn't alter spreading code bit Data rate equal to original spreading code

- Performance similar to FHSS
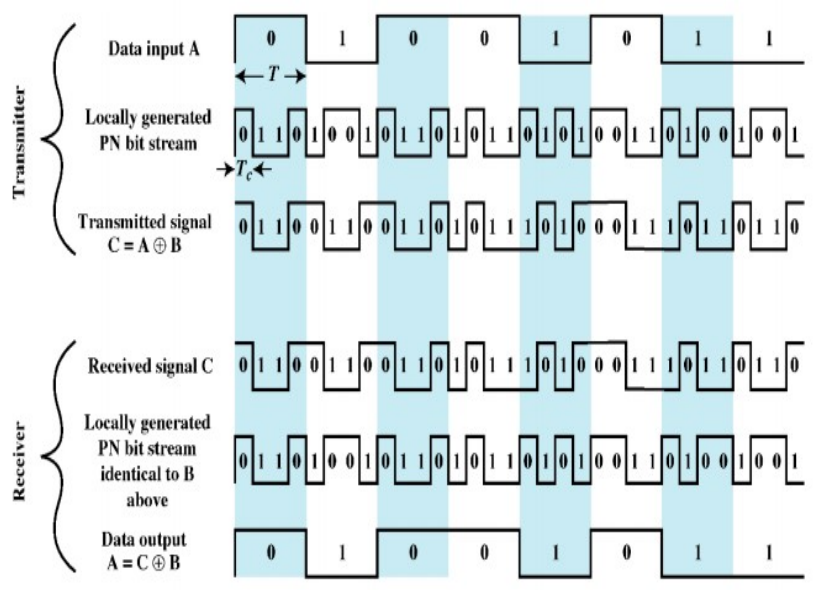

FIG: DSSS EXAMPLE

\section{Principle of DSSS}

Consider the frequency translation of a baseband message (of bandwidth $\mathrm{B} \mathrm{Hz}$ ) to a higher part of the spectrum, using DSBSC modulation. The resulting signal $n$ occupies a bandwidth of $2 \mathrm{~B} \mathrm{~Hz}$, and would typically override the noise occupying the same part of the spectrum. This makes it easy to find with a spectrum analyzer (for example), and so the probability of intercept is high. A local carrier, synchronized with that at

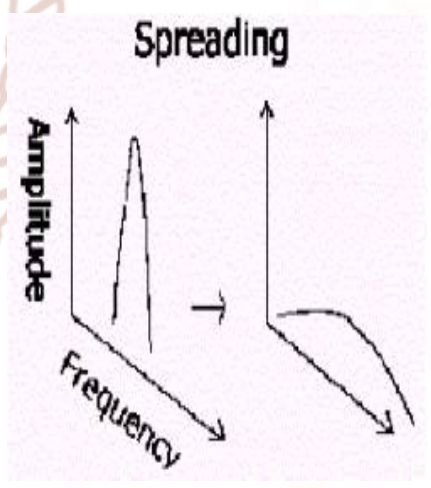
the transmitter, is required at the receiver for synchronous demodulation. The recovered signal-tonoise ratio is $3 \mathrm{~dB}$ better than that measured at its original location in the spectrum. This $3 \mathrm{~dB}$ improvement comes from the fact that the contributions from each sideband add coherently, whereas the noise does not. This can be called a $3 \mathrm{~dB}$ 'processing gain', and is related to the fact that the transmission bandwidth and message bandwidth are in the ratio of $2: 1$

In a spread spectrum system literally thousands of different carriers are used, to generate thousands of DSBSC signals each derived from the same message. 
These carriers are spread over a wide bandwidth (much wider than $2 \mathrm{~B} \mathrm{~Hz}$ ), and so the resulting DSBSC signals will be spread over the same bandwidth. If the total transmitted power is similar to that of the single DSBSC case, then the power of an individual DSBSC in the spread spectrum case is thousands of times less.

\section{Processing gain:}

To achieve most of the claims made for the spread spectrum it is necessary that the bandwidth over which the message is spread be very much greater than the bandwidth of the message itself. Each DSBSC of the DSSS signal is at a level below the noise, but each is processed by the synchronous demodulator to give a $3 \mathrm{~dB}$ SNR improvement. The total improvement is proportional to the number of individual DSBSC components. In fact the processing gain of the system is equal to the ratio of DSSS bandwidth.

\section{Spreading and despreading}

The rapid phase transition $T_{c}$ (chip rate) signal has a larger bandwidth given that the rate is greater $R_{c}$ (without changing the power of the original signal) and behaves similar to noise in such a way that their spectrums are similar for bandwidth in scope. In fact, the power density amplitude of the spread spectrum output signal is similar to the noise floor. The signal is "hidden" under the noise.

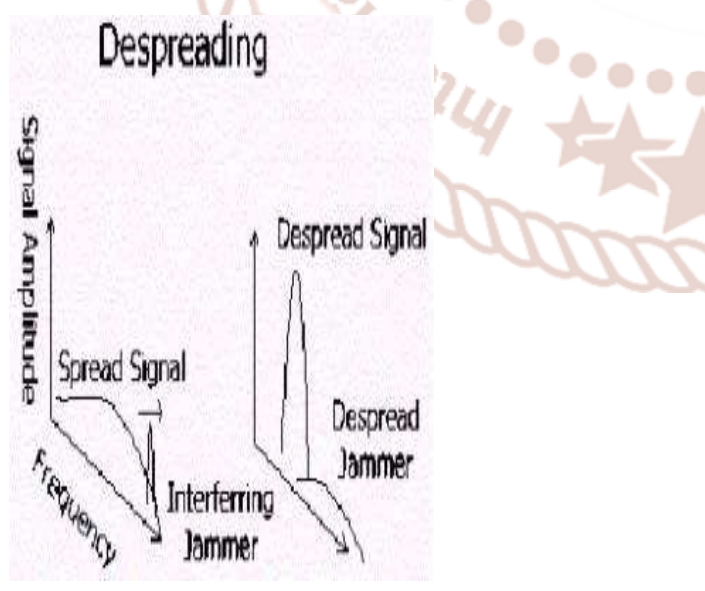

To get the signal back, the exact same high bandwidth signal is needed. This is like a key, only the demodulator that "knows" such a key will be able to demodulate and get the message back. This "key" is in fact a pseudo random sequence (rapid phase transition) also known as pseudo noise (PN). These sequences are generated by $\mathrm{m}$-sequences.

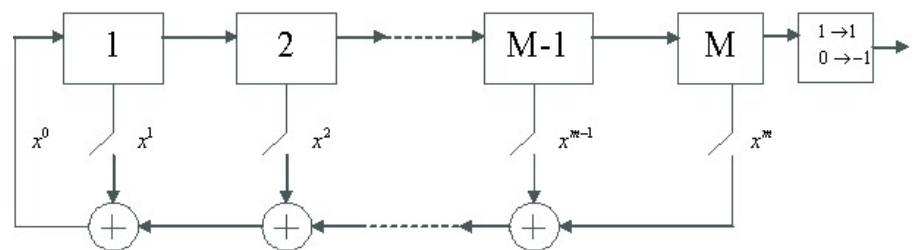

Figure: Shift register structure for $\mathbf{m}$-sequence

Where ' $\oplus$, represent modulo 2 addition. Using this scheme, the initial state is only needed to generate exactly the same sequence of length $2^{m}-1$

\section{BANDWIDTH EFFECTS OF THE SPREADING OPERATION}

Illustrates the evaluation of signal bandwidths in a communicationlink.

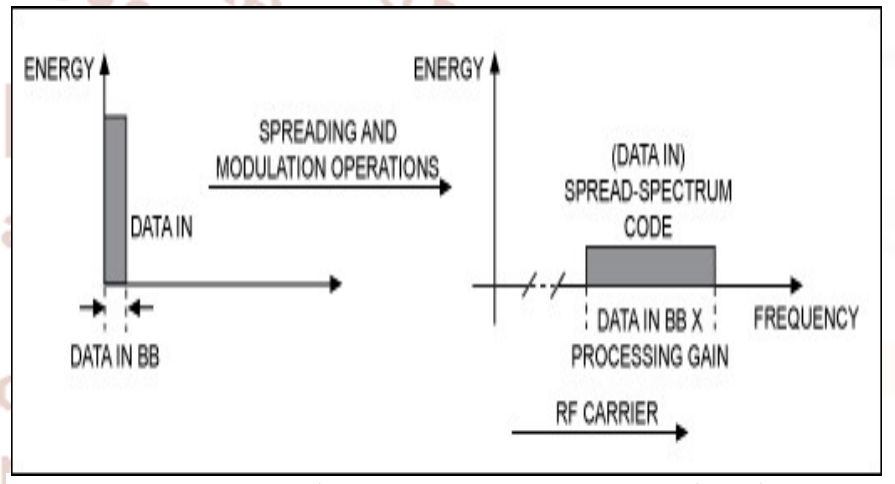

Figure 2. Spreading operation spreads the signal energy over a wider frequency bandwidth.

Spread-spectrum modulation is applied on top of a conventional modulation such as BPSK or direct conversion. One can demonstrate that all other signals not receiving the spread-spectrum code will remain as they are, that is, unspread.

\section{Bandwidth Effects of the Despreading Operation}

Similarly, despreading can be seen in Figure 3. 


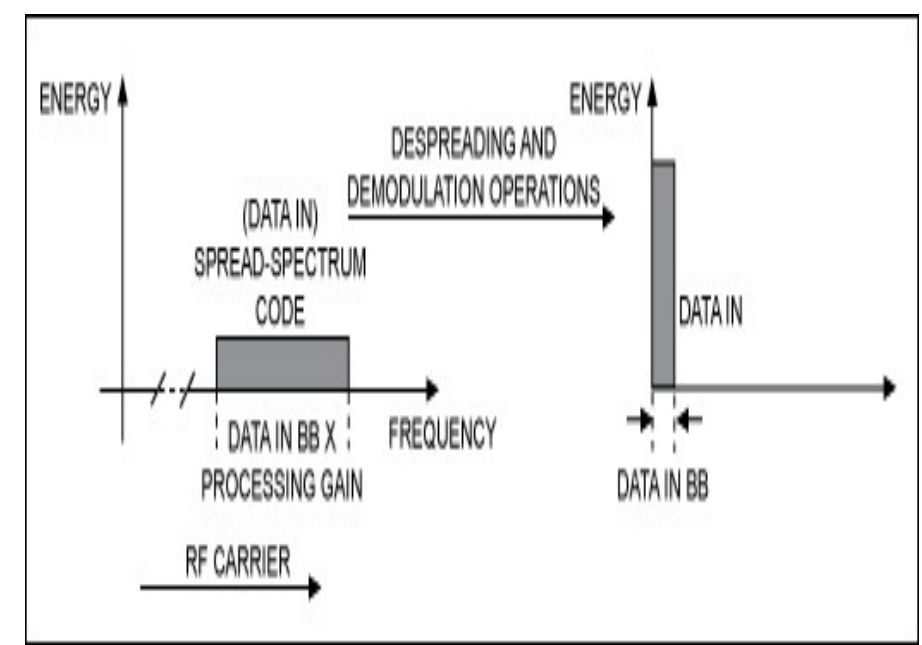

Figure 3. The despreading operation recovers the original

signal.

Here a spread-spectrum demodulation has been made on top of the normal demodulation operations. One can also demonstrate that signals such as an interferer or jammer added during the transmission will be spread during the despreading operation!

\section{Gold Sequences}

Gold sequences help generate more sequences out of a pair of m-sequences giving now many more different sequences to have multiple users. Gold sequences are based on preferred pairs m-sequences.

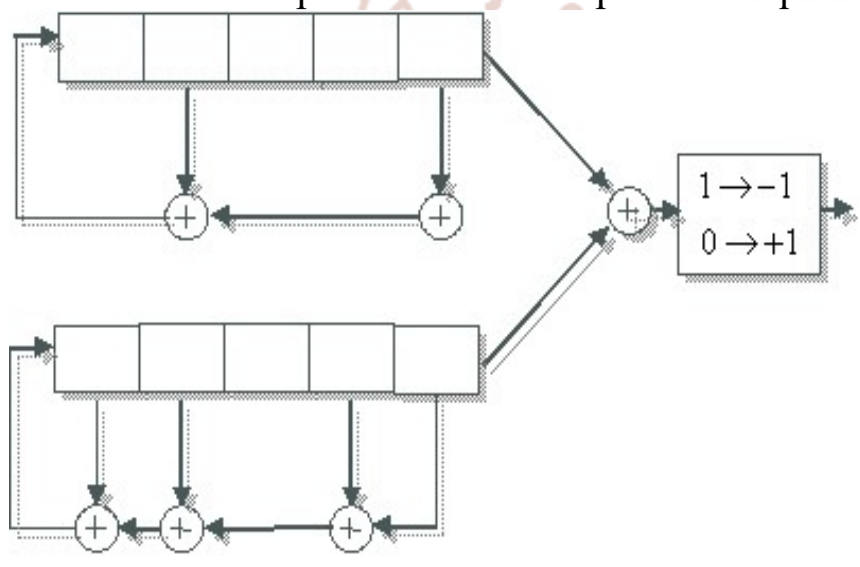

Figure : Example of gold sequence generator

\section{ALGORITHM FOR SIMULATION OF DSSS USING MATLAB}

Step1: Determining of input data to be transmitted and concatenating input sequence.

Step2:Generating of Pseudorandom Noise sequence data

Step3:Implementing of XOR Operation of concatenated signal with PN data to generate hop signal

Step4: Implementation of BPSK Modulation to Hop signal generated

Step5:Implementation of Direct sequence spread Spectrum to modulated BPSK using different carriers

Step6: At receiver implementing reverse operation which include Despreading followed by Demodulation
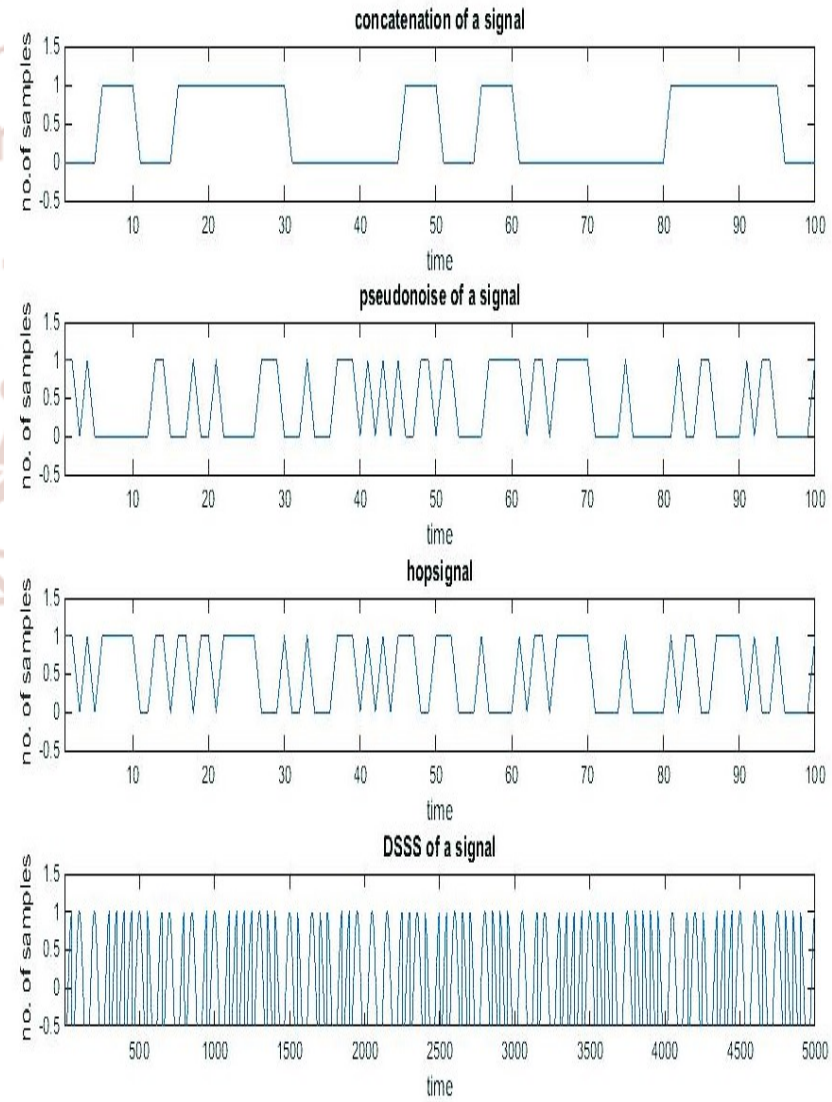


\section{CIRCUIT DIAGRAM OF DIRECT SEQUENCE SPREAD SPECTRUM USING SIMULINK:}

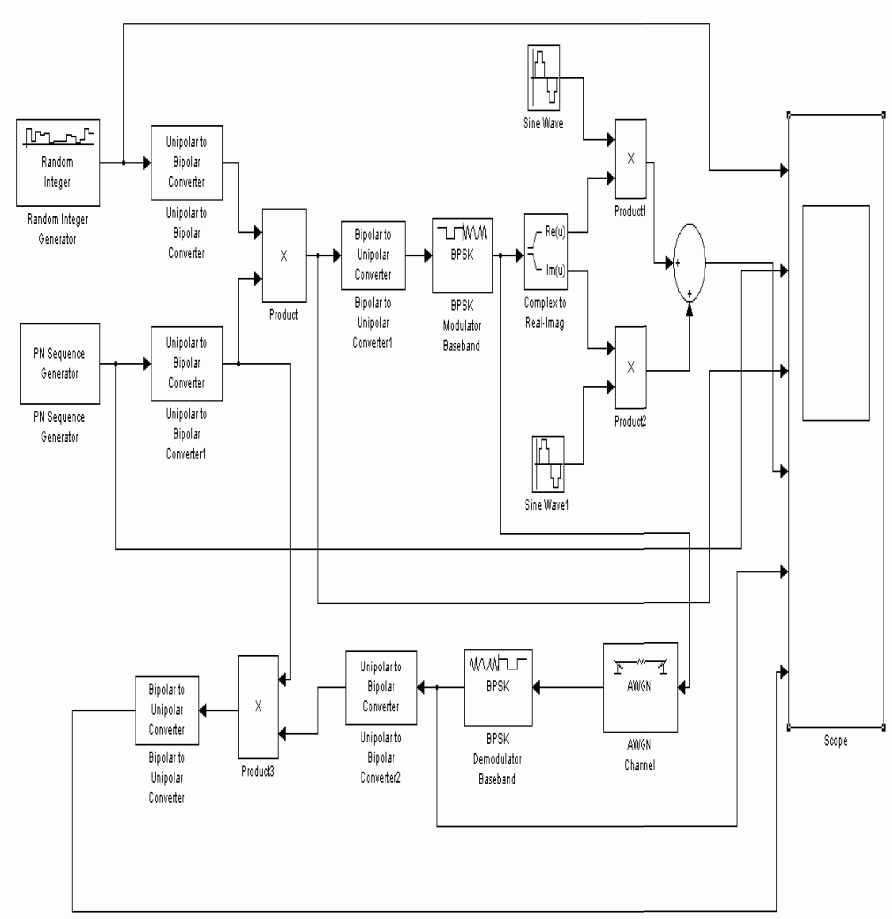

OUTPUT OF DIRECT SEQUENCE SPREAD SPECTRUM USING SIMULINK:

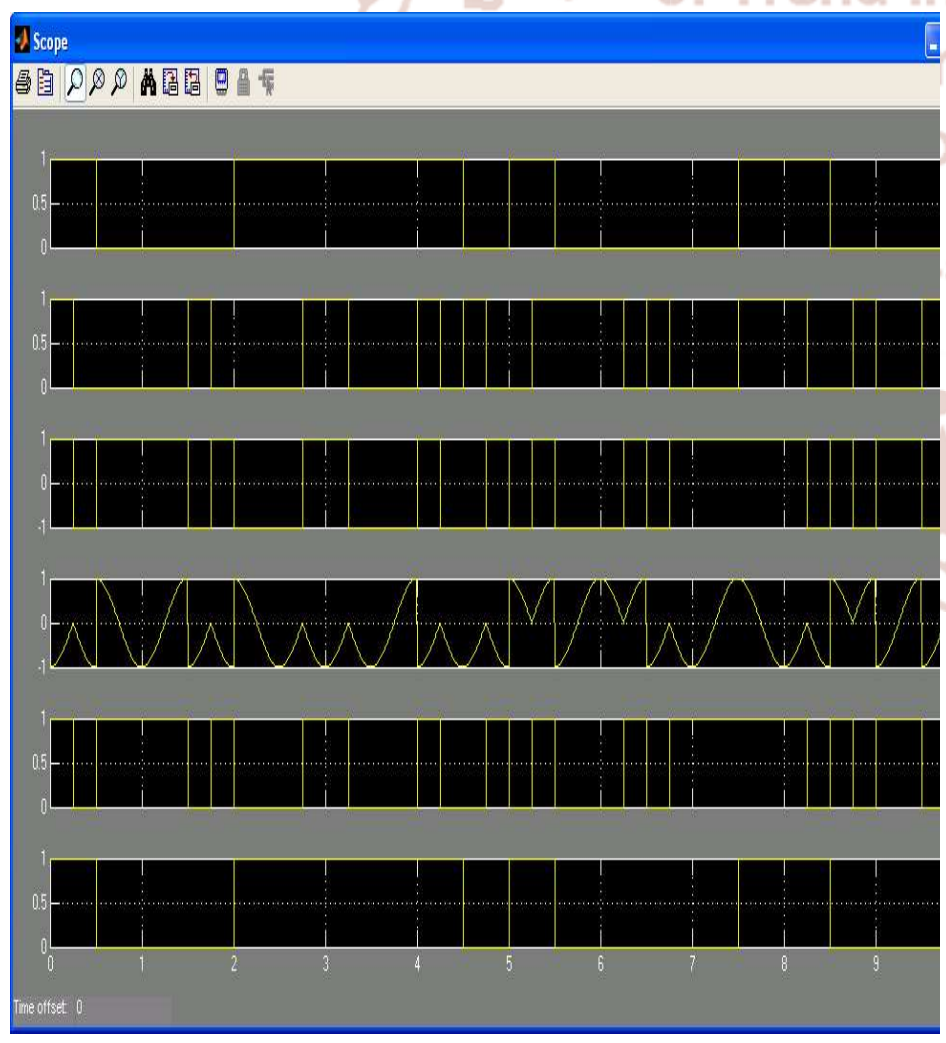

\section{CONCLUSION}

Direct Sequence Spread spectrum is a technique that expands to many different paths, modulation schemes, performance under fading, under interference, increases capacity in CDMA systems.

It provides privacy \& Secure Communication, protection against jamming, better voice quality \& Low susceptibility and can operate longer distances.

\section{References}

1) "Review on Design \& Implementation of DSSSCDMA Transmitter using HDL with Raised cosine filter to minimize ISI" IJCSMC Vol 3,Issue3,March 2014

2) G. L. Stüber, "Principles of mobile communication," Kluwer Academic, Boston 1996.

3) J. G. Proakis, "Digital Communications," 4th Edition, McGraw-Hill Higher Education.

4) T. S. Rappaport, "Wireless communications: principles and practice," Pentrice Hall PTR, N.J., 1996.

5) T: Pratt, C. W. Bostian and J.E. Allnutt, "Satellite Communications," 2nd ed. John Wiley publication, 2002.

6) R: Prasad, T. Ojanperä, "An Overview of CDMA Evolution Toward wideband CDMA," IEEE communications surveys, Vol. 1, No. 1 Q4 1998 